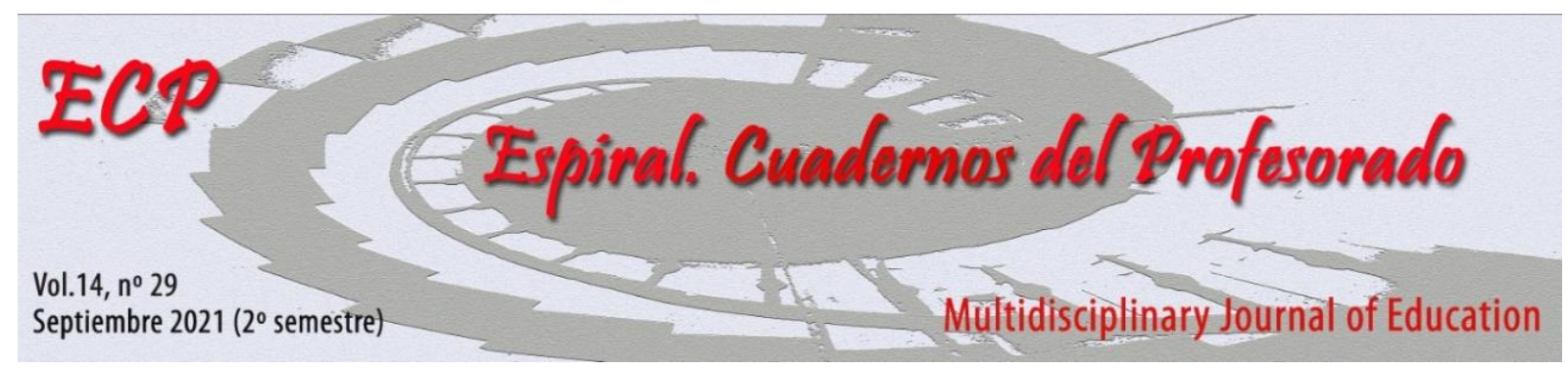

\title{
Epitextos editoriales en la promoción y mediación de los libros de no ficción
}

\section{Publishing houses in the promotion and mediation of non-fiction}

\author{
Manuel Francisco Romero Oliva ${ }^{1}$, Blanca Florido Zarazaga ${ }^{1}$, Hugo Heredia Ponce ${ }^{1}$ \\ ${ }^{1}$ Universidad de Cádiz, España
}

\begin{abstract}
Resumen
El presente artículo consiste en una investigación de corte cualitativo, más concretamente un análisis del cuerpo editorial que trabajan la línea de no ficción para un único objetivo: examinar los epitextos editoriales de cara la promoción y mediación de este tipo de publicaciones. Para tal fin, se han desarrollado tres fases: referencia de expertos para obtener información sobre el problema objeto de estudio; elaboración de una cartografía lectora basado en los libros ilustrados informacionales tanto para áreas lingüísticas como áreas no lingüísticas y tomando los epitextos como criterio de composición; y, por último, una propuesta de dinamización de estos libros utilizando los epitextos, en este caso una reseña, para así atender al carácter interdisciplinar de la competencia en comunicación lingüística -en adelante, CCL-. Así pues, la escuela se encuentra ajustada a la sociedad en la que nos ubicamos teniendo como resultado la formación de ciudadanos socialmente competentes y críticos desarrollando habilidades para leer en formato impreso y digital.
\end{abstract}

Palabras clave: Libro de no ficción; Epitextos editoriales; Competencia en comunicación lingüistica; Áreas lingüísticas y áreas no lingüísticas.

\begin{abstract}
The present article consists of qualitative research, more specifically an analysis of the editorial body that works the non-fiction line for a single objective: to examine the editorial epitextes in order to promote and mediate this type of publications. To this end, three phases have been developed: reference by experts to obtain information on the problem under study; elaboration of a reading cartography based on the informational non-ilustrated books for both linguistic areas and non-linguistic areas being the epitextes as a composition criterion. Finally, an example of how it is possible to dynamize these books by using the epitextes, in this case a review, in order to take into account, the interdisciplinary character of the competence in linguistic communication -from now on CCL-. Thus, the school is adjusted to the society in which we are located, having as a result the formation of competent and critical citizens developing skills for reading in printed and digital format.
\end{abstract}

Keywords: Non-fiction books; Editorials; Competence in linguistic communication; Linguistic areas and nonlinguistic areas.

Fecha de recepción: 26/01/2021

Fecha de aceptación: 12/03/2021

Correspondencia: Hugo Heredia Ponce, Universidad de Cádiz, España Email: hugo.heredia@uca.es 


\section{Introducción}

En primer lugar, sería importante señalar que uno de los retos de la escuela se centra en la enseñanza de la lectura $y$, como consecuencia, en la formación de lectores, puesto que juega un papel indispensable en el desarrollo integral de la persona y en el aprendizaje durante toda la vida (Maina, 2018). Tanto es así que el dominio de la lectura asegura el acceso al conocimiento, no ignorando que palabras como éxito o fracaso escolar están estrechamente relacionadas con este tipo de actividad (Romero \& Trigo, 2015): "un deficiente aprendizaje lector y una mala comprensión de lo leído abocan a los alumnos y las alumnas al fracaso escolar y personal" (Instrucciones de 24 de julio de 2013, p.1). Por esta razón, se hace necesario consolidar la relevancia de la competencia lingüística y los hábitos lectores desde todas las áreas lingüísticas (en adelante, AL) y áreas no lingüísticas (en adelante, ANL) etapas curriculares (Herrera, 2009; Ballester \& Ibarra, 2016).

Sin embargo, el estudio de la lectura se percibe desde una perspectiva amplia y holística, pues así como cualquier otro elemento educativo se considera un ecosistema que para su equilibrio no solo converge aspectos circundantes de los jóvenes lectores, sino también agentes extrínsecos a ellos, donde incluimos a los llamados mediadores no institucionales (familias, iguales, librerías, editoriales, medios de comunicación convencionales y alternativos, como YouTube, booktubers, wattapad, instagramers, etc.). (Romero, et al. 2020). Conectamos con la idea de Bronfenbrenner (1987) quien centra su modelo en cuatro subniveles ecológicos presentando una interdependencia mutua: microsistema, mesosistema, exosistema y macrosistema. Será en el ámbito del mesositema donde ubiquemos la influencia de las editoriales dentro de los otros entornos inmediatos donde la persona interactúa se centran en el ámbito municipal, su ciudad, y los servicios que le ofrecen: bibliotecas, librerías, centros comerciales y de ocio, clubes y asociaciones recreativas... conformando una serie de posibilidades culturales favorecedoras del acceso a la cultura y un medio de compartirla (Trilla, 2005). Es decir: la lectura y lectores cambian y se transforman desde una dimensión cultural y social y, por tanto, si se indaga en innovación, evitando el clasicismo y tradición, se deduce que existen nuevos momentos, nuevas formas de leer y nuevos consumos lectores.

Ante esto, se deduce que la lectura es una realidad compleja y sus prácticas sociales no pueden desarraigarse de las coordenadas espacio-tiempo de la comunidad en la cual se dinamiza, pues esta ha de concebirse desde la noción integradora de modernidad líquida (Bauman, 2019) donde prevalece su carácter mutable y dinámico. Así pues, todos estos aspectos nos hacen reflexionar sobre cómo los profesionales de la educación pueden afrontar el reto de la formación lectora en las aulas actuales. Se presenta aquí una visión integradora de centro desde el Proyecto Lingüístico de Centro (PLC) (Gómez, 2013; Trujillo \& Rubio, 2014) como una piedra angular para afrontar esta problemática desde las distintas líneas de actuación que ofrece (Trujillo, 2010); será concretamente la de formación de lectores la que tomará relevancia en este estudio. Este planteamiento ofrece la posibilidad de trabajar la competencia en comunicación lingüística (Fabregat, 2016), contribuyendo no solo al desarrollo de la comprensión lectora sino a los planes de lectura y biblioteca desde una visión de centro (Santos, 2017), en donde se integren las áreas linguísticas (AL) y no lingüísticas (ANL) (Fabregat, 2018; Romero \& Trigo, 2018).

Por otro lado, las propuestas de nuevos enfoques metodológicos - trabajar desde la integración curricular y metodologías por proyectos- conlleva el desarrollo de la interdisciplinaridad mediante propuestas de trabajos como los centros de interés, la investigación del medio natural, aprendizaje basado en problemas, proyectos globales (Zabala \& Arnau, 2014). En todos ellos, se intenta ampliar la curiosidad del estudiante mediante la investigación para acercarse al entorno y comprender la realidad (Lorenzo, 2015). Para tal fin, se debe tener en cuenta que la acelerada evolución de las Tecnologías de la Información y Comunicación -TIC- ha generado nuevos formatos, nuevos medios, nuevas formas de lectura y nuevos géneros (Cordón, 2018). En este panorama, el libro-álbum ilustrado de no ficción puede adquirir un protagonismo centrado en la integración de ANL ya que, además, se trata de un género que ha tenido gran repercusión a partir de los años noventa en el mercado editorial, fundamental en el desarrollo de la competencia lecto-literaria del receptor del siglo XXI (Tabernero, 2011). 
Por todo ello, nuestra investigación se encuadra dentro del proyecto Formar lectores en la sociedad digital desde los libros de no ficción (RTI2018-093825-B-I00), perteneciente Proyectos I+D+i de la convocatoria de Retos Investigación de la Agencia Estatal de Investigación, y se centra en una de las aristas que conforman dicho proyecto: los nuevos formatos editoriales del género "libro de no ficción" como medios de máximo interés para la formación de lectores competentes en el modelo analógico y digital al singularizarse por integrar la materialidad propia del libro con estrategias visuales e hipervinculares características de los soportes virtuales, en nuestro caso, los epitextos editoriales. Por tanto, el objetivo que se ha determinado es analizar los epitextos editoriales de cara a la promoción y mediación de los libros informacionales en la creación de lectores críticos y competentes en la escuela actual. Las diferentes cuestiones que se pretenden averiguar para construir conocimiento sobre el fenómeno objeto de estudio son: por una parte, por qué este tipo de libros se considera un género conveniente para el lector del sigo XXI; en segundo lugar, qué lecturas y qué estrategias, en nuestro caso epitextos lectores, publicitan las editoriales de cara a la promoción del libro; y, por otra parte, cómo los libros de no ficción llegan a convertirse en un elemento fundamental para la integración de AL y ANL desde la creación de cartografías lectoras en donde se integren todas las áreas del currículo.

\section{Método}

\section{Diseño de investigación}

Tomando como referencia las tres eras paradigmáticas de la investigación educativa que establece Soltis (1984 citado por Rodríguez-Gómez, 1999), la finalidad que se persigue en este estudio se circunscribe en un planteamiento interpretativo de corte cualitativo al intentar comprender, en situaciones particulares, el significado de los epitextos editoriales en la promoción y mediación de los libros informacionales. Además, esta perspectiva queda matizada en el enfoque etnográfico porque a través de este se busca sacar a luz el modo en que, en este caso, los sujetos informantes conocen este tipo de libros y su panorama editorial. En otras palabras, el investigador interpretativo se basa en el conocimiento de estos para poder interpretar la experiencia y generar nuevas formas de actuar en las aulas actuales.

\section{Participantes}

En esta investigación, la población objetiva $(\mathrm{N})$, también denominada según McMillan \& Schumacher (2005) diana o universo es, por una parte, la investigadora principal del proyecto donde se integra esta investigación y, por otra parte, un grupo de expertos compuesto por tres miembros del proyecto: dos de la Universidad de Cádiz y una de la Universidad de Zaragoza. Con la idea de poder abrir horizontes sobre el fenómeno objeto de estudio y obtener así una visión más global y precisa, se ha entrevistado a los informantes a través del programa Google Meet, debido a la situación originada por la Covid-19 pero atendiendo a las nociones claves de la etnografía tradicional de cualquier trabajo de campo.

\section{Fases de la investigación}

Esta investigación toma como referencia las fases que establecen Rodríguez, et al (1999) respecto a la investigación cualitativa: preparatoria, trabajo de campo, analítica e informativa. Sin embargo, el hecho de no poder acceder al campo objeto de estudio -en un principio el centro educativopor el cierre temporal de los colegios ha tenido como desencadenante principal que este trabajo se haya establecido en torno a tres etapas según las tres dimensiones que establecen Trigo, et al. (2019):

\section{Fase 1: preparatoria. Punto de arranque: la voz de los especialistas}

Para la elaboración en la cartografía lectora - fase 2-y el desarrollo de un modelo de actuación para la dinamización de los libros ilustrados de no ficción desde los epitextos editoriales — fase 4-, tiene lugar una toma de decisiones que se fundamenta en la voz de referencia de expertos. O sea, se ha obtenido información de diferentes fuentes para comparar situaciones, percibir contrariedades y semejanzas, así como escuchar las diferentes valoraciones sobre el fenómeno objeto de estudio; tal y como señalan Rodríguez, et al. (1999). 


\section{Fase 2: trabajo de campo. Análisis documental de epitextos editoriales}

Para elaborar una cartografía se tomaron como referencias las editoriales relevantes hispanohablantes en álbumes y libros ilustrados de no ficción. En nuestro caso, la conversaciones con los expertos, sirvieron de referencia para la propuesta de un itinerario lector para Educación Primaria donde las AL y ANL, según Romero y Trigo (2015), estuvieran representadas desde una visión interdisciplinar de la CCL. Asimismo, se especifican los contenidos curriculares que, según la Orden 17/03/2015, por la que se desarrolla el currículo correspondiente a la Educación Primaria en Andalucía, pudieran estar relacionados con los libros propuestos. Sin embargo, estas propuestas curriculares son asumibles, por su carácter genérico, por cualquiera de las comunidades autonómicas ya que se vinculan a materias más que a contenidos específicos. Finalmente, se incluyen un enlace (link) que nos llevará al epitexto del libro (blogs, reseñas, booktrailers...).

\section{Fase 3: analítica. Actuaciones para considerar la CCL desde su carácter interdisciplinar}

Una vez que vez que se ha obtenido toda la información necesaria en el análisis editorial, se elabora un Plan de Lectura y Biblioteca como oportunidad para abordar futuros planes de mejora. Dos son los momentos clave en la investigación: el estudio de editoriales de referencia basada en los libros informacionales y la elaboración de una cartografía lectora para ejecutar un itinerario lector basado en este género en el que se incorporan AL y ANL.

\section{Fase 4: informativa. Estrategias de visibilización del PLC}

Después de que se ha elaborado la cartografía lectora que configurará la supuesta biblioteca escolar, se desarrollará una propuesta para dinamizar un libro utilizando los epitextos (booktrailer, reseñas, blog...) con carácter interdisciplinar. Esta se podrá integrar en el Plan de Lectura y Biblioteca y así trabajar los libros informacionales tanto en las AL como en las ANL. De esta forma, se consigue contribuir al desarrollo de la CCL desde una visión sistémica de centro, tomando como referencia los libros de no ficción.

\section{Estrategias de recogida de información}

Uno de los retos de este trabajo de investigación era acercarse a los hechos sobre el fenómeno objeto de estudio (Campoy \& Gomes, 2009) y, por esta razón, para acometer el tratamiento de los datos se ha hecho uso de distintas técnicas. Es en la primera fase cuando se llevó a cabo una entrevista etnográfica para la obtención de información (Vázquez \& Angulo, 2003). Para ello, se estableció previamente un guion en el que se incluye las preguntas de investigación - Tabla 1-.

Tabla 1

Relación entre dimensiones y cuestiones

\begin{tabular}{ll}
\hline \multicolumn{1}{c}{ Categorías/Dimensiones } & \multicolumn{1}{c}{ Preguntas } \\
\hline Libro de no ficción & $\begin{array}{l}\text { ¿Cuál es el motivo por el que el libro de no ficción es un género } \\
\text { conveniente para el lector del siglo XXI? }\end{array}$ \\
& $\begin{array}{l}\text { ¿Los libros informacionales se consideran cruciales para la } \\
\text { integración de AL y ANL? }\end{array}$ \\
\hline Panorama editorial & $\begin{array}{l}\text { ¿Cuáles son las editoriales de mayor prestigio que están trabajando } \\
\text { más en la línea de no ficción? }\end{array}$ \\
\hline
\end{tabular}

A continuación - fase 2-, con la finalidad de analizar el cuerpo editorial de esta biblioteca se realizó, siguiendo a López (2002) y Rodríguez-Gómez (2011), un análisis de documentos o contenido para poder así elaborar y confeccionar una cartografía lectora basado en los libros de no ficción integrando AL y ANL. 


\section{Resultados y discusiones}

\section{Fase 1. Punto de arranque: la voz de los especialistas}

Con el fin de obtener una mejor comprensión del problema objeto de estudio, se han establecido diferentes categorías relacionadas con las cuestiones de investigación que han sido planteadas que, como se ha indicado anteriormente, serán dos: el libro de no ficción y el panorama editorial. A continuación, se expone el análisis de ambas categorías justificándolo con los propios comentarios de los sujetos informantes.

Análisis de la categoría 1. Libros ilustrados de no ficción

- ¿Cuál es el motivo por el que el libro de no ficción es un género conveniente para el lector del siglo XXI?

Según INF_Inv2 ${ }^{l}$, a pesar de que la hipertextualidad es una característica propia de las pantallas -modelo digital-, una similitud entre este y el analógico es la estructura que presentan. La lectura en este primer modelo mencionado ofrece la necesidad de precisar la información que se recoge en estos libros ya que "el niño necesita coger un libro, ir página por página siguiendo la guía que el autor le va dando, la secuenciación, jerarquización de conocimientos, selección" (INF_Inv1). Además, un aspecto imprescindible es su componente artístico; esto es la relación entre palabra e imagen (INF_Inv4). Esto se debe a que creemos que observar es un acto tan habitual que no nos paramos a reflexionar sobre nuestras percepciones. Es decir: "el concepto artístico-estético-físico y la imagen ayuda a ese lector que está muy entretenido en las redes donde es todo muy rápido y no da tiempo a fijarse en nada" (INF_Inv1).

- ¿Los libros informacionales se consideran cruciales para la integración de AL y ANL?

Para dinamizar el libro de no ficción en el aula se puede optar por diversas estrategias -ya sea de lectura o del producto-, pues se pretende convertir el acto lector en un momento donde confluya la lectura individualizada con la compartida (INF_Inv4). Es el caso de la lectura en redes sociales y el apoyo de herramientas digitales como el booktrailer o el booktuber, según INF_Inv3. En resumen, "es una forma de leer compartiendo para generar la curiosidad, para interpretar el mundo y para acceder a nueva información; esto es hipervincular a nuevos contenidos" (INF_Inv4). Por consiguiente, este tipo de lectura contribuye al desarrollo interdisciplinar de la CCL (INF_Inv4). Además, una de las ventajas con las que cuentan es que es posible trasladarte al mundo exterior: "tiene una parte contextual de la que no dispone el libro de ficción" (INF_Inv1).

Análisis de la categoría 2. Panorama editorial

- ¿Cuáles son las editoriales de mayor prestigio que están trabajando más en la línea de no ficción?

Tanto el INF_Inv1 como INF_Inv4 afirman que la editorial Juventud se convierte en una de sus referencias, pues observa que "sabe muy bien a qué publico va dirigido, distingue entre temas y edades, incluso entre chicos y chicas" (INF_Inv1). Por otro lado, el INF_Inv3 prefiere los libros del Zorro Rojo ya que considera que todos sus libros vienen acompañados de un booktrailer como epitexto virtual público. Otras editoriales relevantes en este tema, según el INF_Inv1, son: MaevaYoung, Takatuka, Iamiqué, Tecolote, Amanuta, CocoBooks, A Buen Paso, Nube Ocho, Wonder Ponder y Media Vaca, entre otras. Estas dos últimas editoriales mencionadas contribuyen al desarrollo del pensamiento político y crítico de los primeros lectores, pues "en vez de infantilizar, lo que hace es madurar en temas de la sociedad importantes" (INF_Inv4).

Fase 2. Análisis y selección documental de epitextos editoriales

Como indican Lluch, et al. (2015), los epitextos públicos virtuales u online adquieren su valor no solo para promocionar un libro, un autor o un tema, sino también para añadirles nuevos significados en la cultura y en la sociedad. La autora distingue los siguientes (Lluch, et al. 2015, p. 800):

\footnotetext{
${ }^{1}$ Para guardar el anonimato de los informantes se ha utilizado INF_Inv $+n^{\circ}$. 
- Blogs y foros de lectores: conversaciones creadas en un espacio virtual de comunicación que generan espacios donde se crean lazos de pertenencia a un grupo a través de unas características comunes de la lectura

- Book-trailers o trailers de libros: el book-trailer es un instrumento de promoción de un libro en formato de vídeo que emplea técnicas similares a las que utiliza el tráiler cinematográfico con la peculiaridad de que se difunde a través de las redes sociales. Se define como un modo de promoción que tiene que ver con un lector, el del siglo XXI, que se desenvuelve de una forma natural en las redes sociales y recibe la información a través de soportes multimedia que integran palabra, imagen y sonido de naturaleza hipertextual.

- Los prescriptores de libros como críticos literarios, profesores universitarios o revistas especializadas comparten su espacio con los booktubers y las redes sociales.

Nuestra opción - Tabla 2 - consistió en confeccionar una cartografía de libros ilustrados de no ficción, a modo de ejemplo, en el curso de $6^{\circ}$ de Educación Primaria donde se integrarán áreas y materias del currículo con un libro informacional específico y el epitexto virtual que ofrecen las editoriales.

Tabla 2

Cartografía de libros de no ficción. $6^{\circ} \mathrm{EPO}$

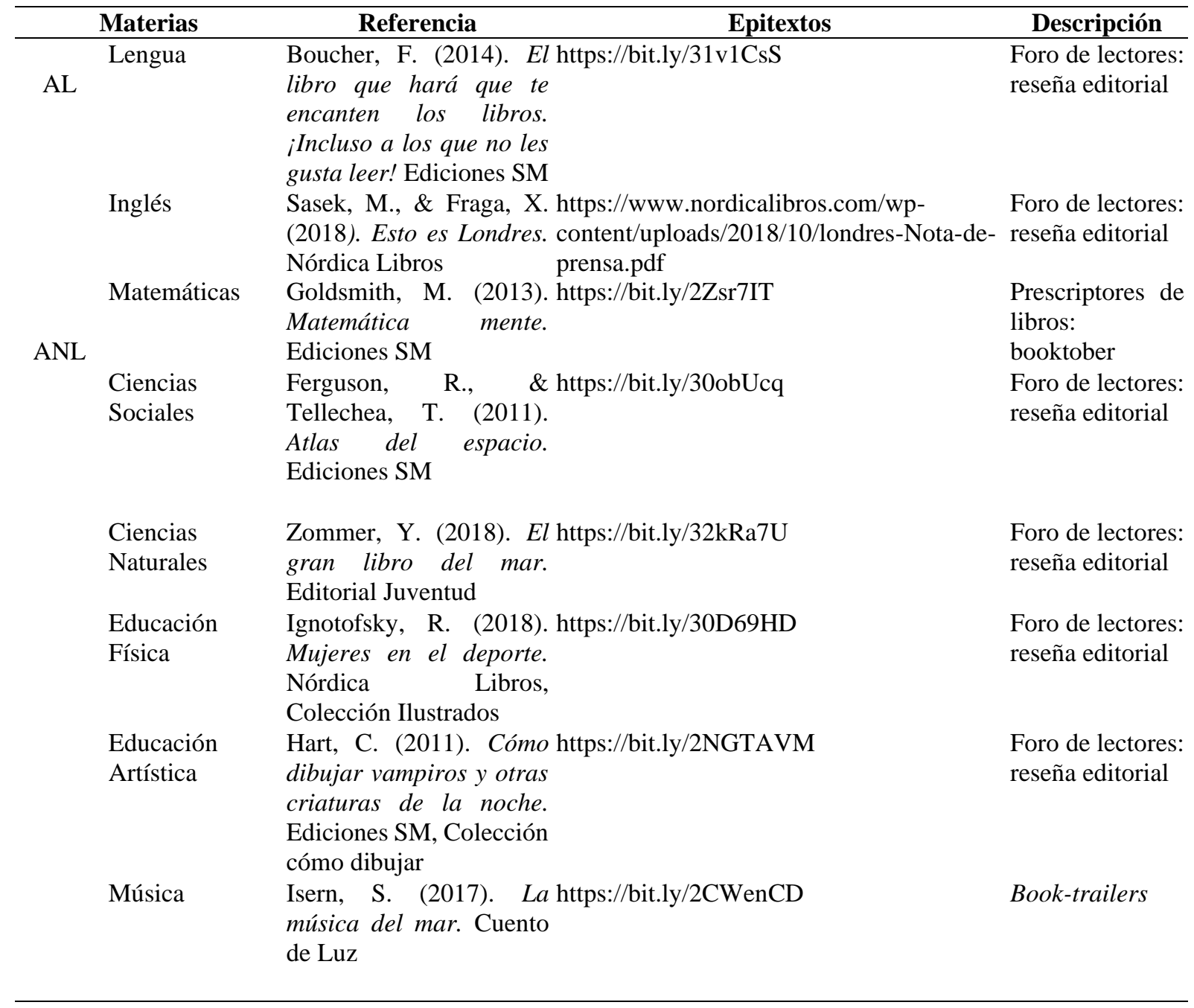

Fuente: Elaboración propia 
En definitiva, la ampliación del concepto de epitexto virtual u online a los nuevos escenarios escolares y las prácticas sociales de lectura aporta una línea de trabajo imprescindible para la promoción del libro y la formación de lectores críticos desde la contribución de todas las áreas y materias del currículo.

Fase 3. Actuaciones para considerar la CCL desde su carácter interdisciplinar

En la elaboración de la cartografía lectora se ha tomado en consideración las editoriales más significativas en el panorama hispanohablante en álbum y libro ilustrado de no ficción, siguiendo las aportaciones de los informantes entrevistados en la fase 1. Los criterios de conformación de la cartografía lectora fueron los siguientes:

- Columna 1: área de conocimiento y ámbito del área -AL o ANL-

- Columna 3: contenidos según Instrucción 8/2020 del 15 de junio

- Columna 4: referencia bibliográfica de la editorial del libro

- Columna 5: epitexto del libro (booktrailer, reseñas, blogs...)

Tabla 3

Cartografía de libros de no ficción. $6^{\circ}$ EPO

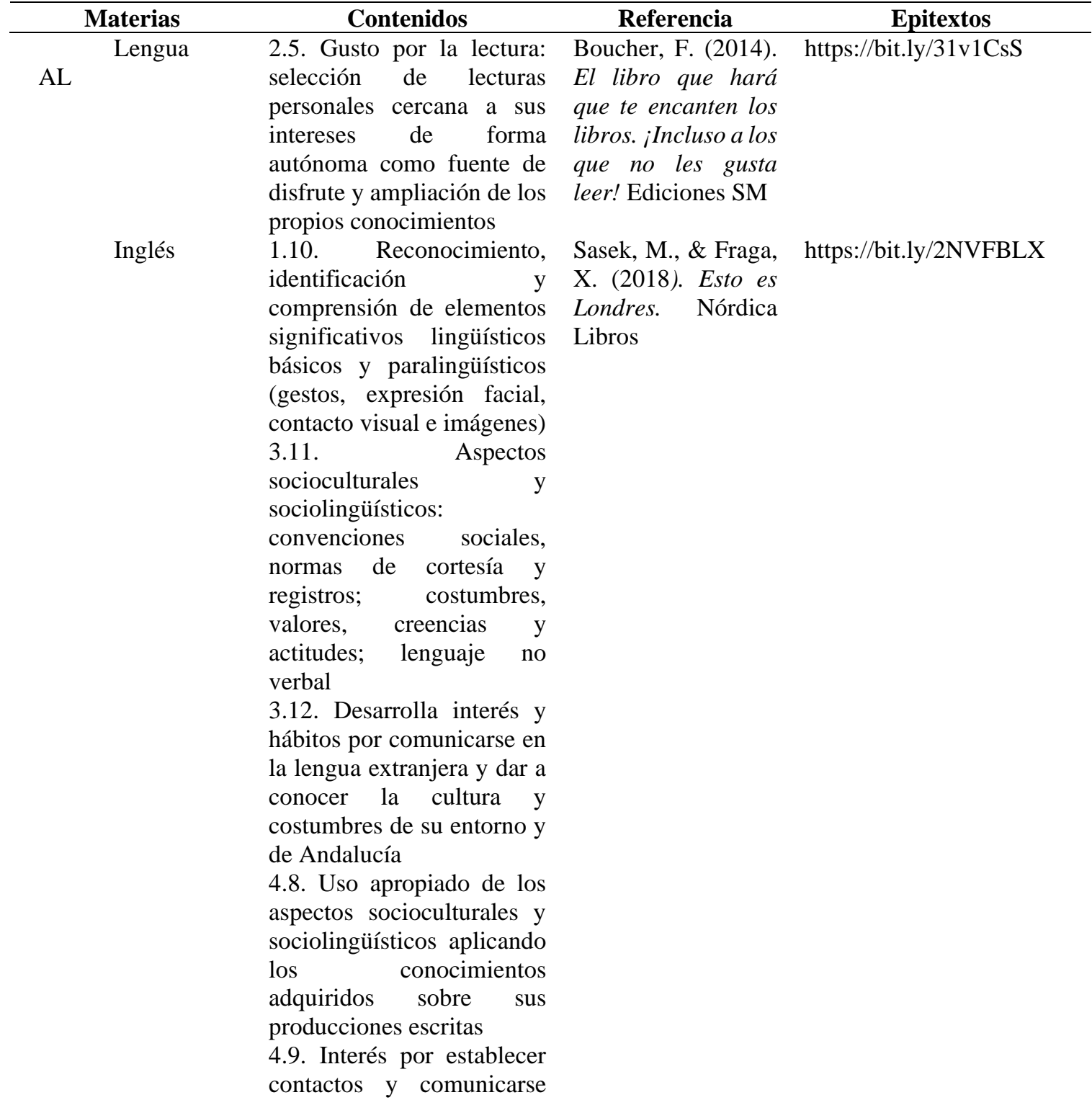

Espiral. Cuadernos del Profesorado | ISSN 1988-7701 | 2021, 14(29), 41-55 


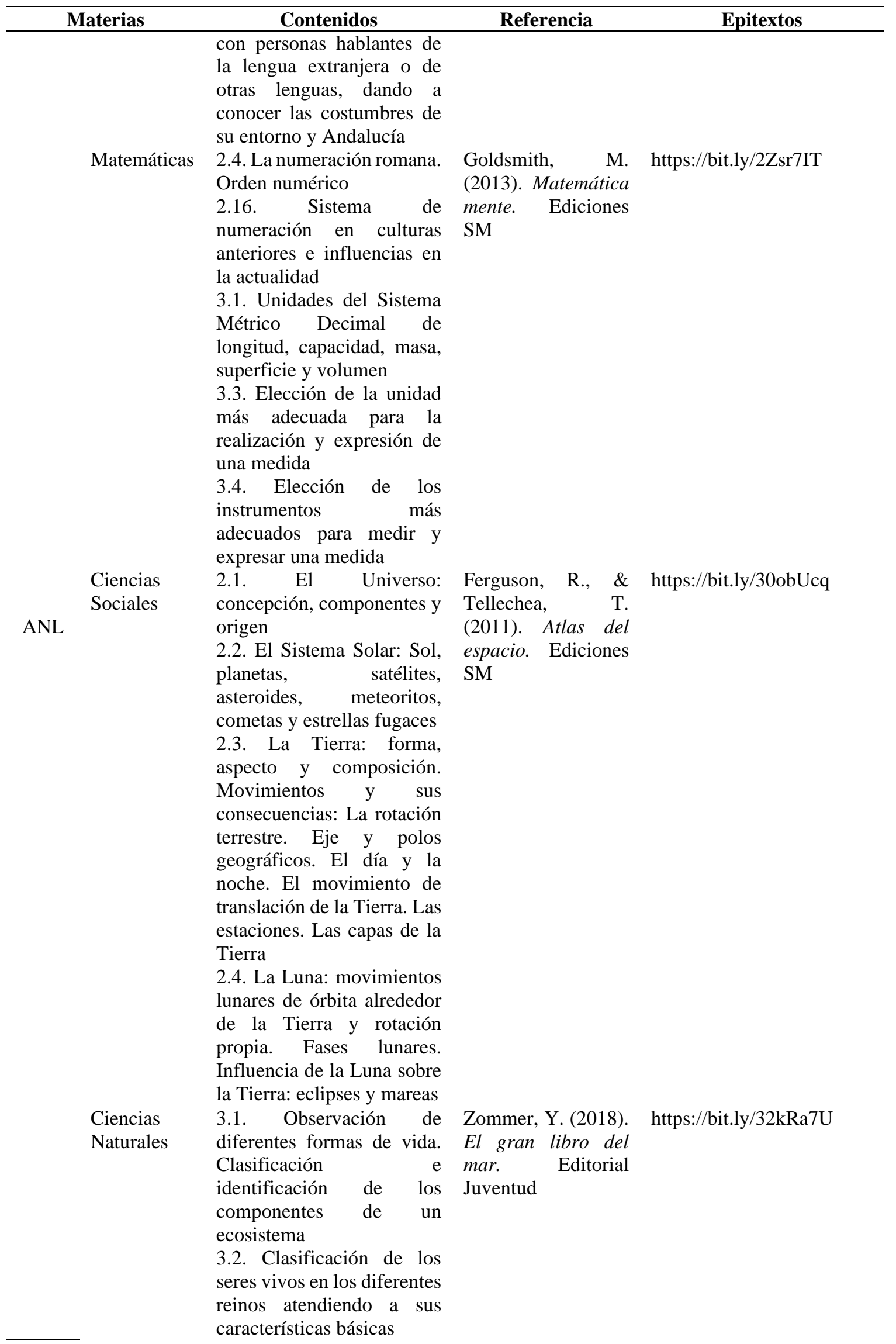




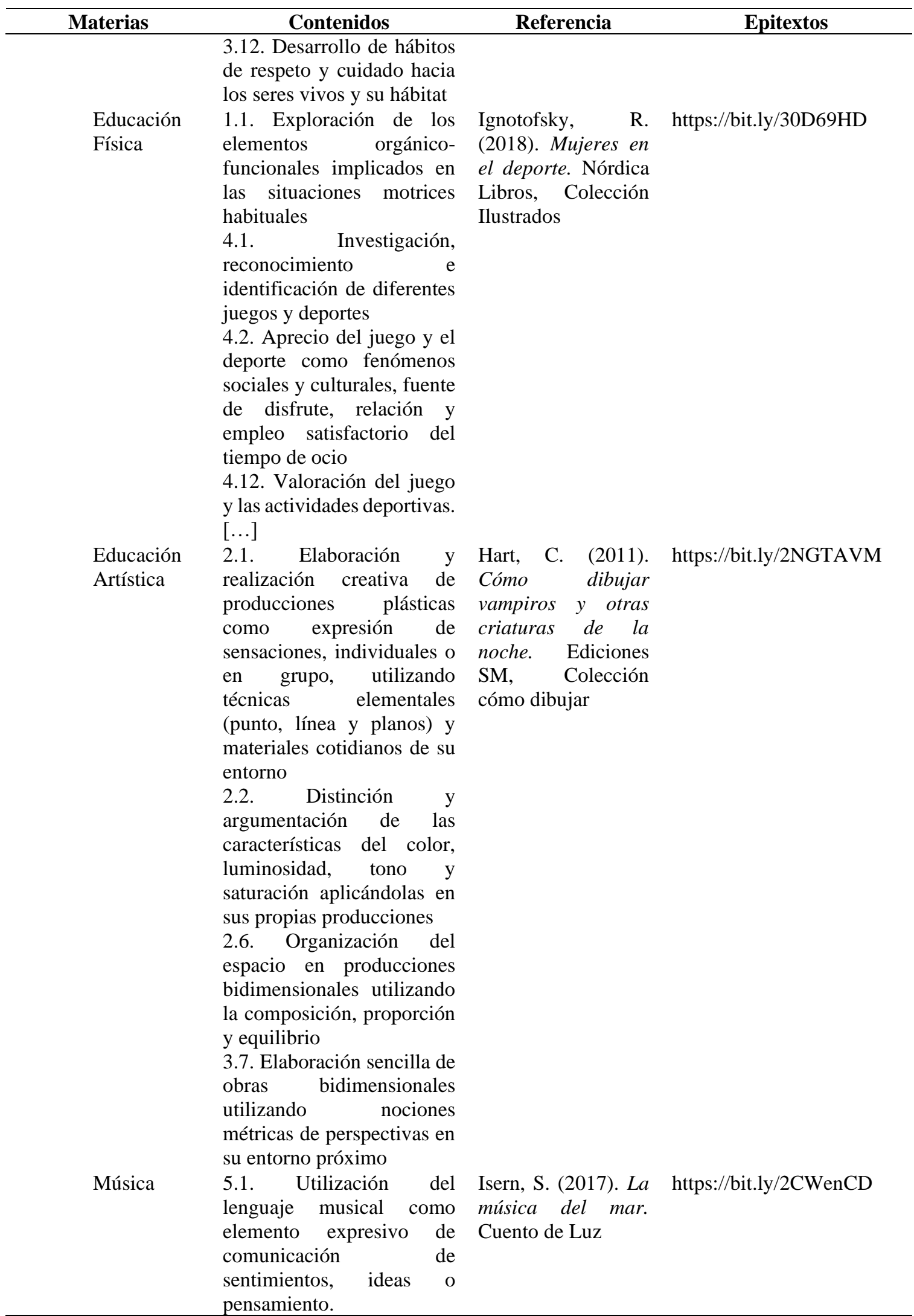

Fuente: Elaboración propia

En la propuesta dirigida al $6^{\circ}$ curso de Educación Primaria - Tabla 3-, el libro de no ficción para lengua castellana y literatura señala distintas ventajas de la lectura en comparación con otros

Espiral. Cuadernos del Profesorado | ISSN 1988-7701 | 2021, 14(29), 41-55 
hobbies justificando de forma dinámica y divertida las razones por las que leer resulta una actividad placentera. En el caso de inglés, se intenta transportar al alumnado a Londres, ya que a través de sus páginas es posible descubrir sus lugares icono, así como los aspectos más extraños de sus residentes. Del mismo modo, no solo aprenderán mates desde el punto de vista práctico, sino que además se atenderá a su carácter histórico y numérico con las diferentes actividades que se muestran. En la asignatura de ciencias sociales, se viaja por el espacio con toda la información que el libro proporciona: Sistema Solar en tres dimensiones, mapas del espacio que indican la ubicación de cada planeta y páginas deslizables sobre la Luna, el Sol, la Vía Láctea y el universo. Asimismo, cada página del libro explica una especie en concreto -pulpos, medusas, kril...- o sobre cultura general sobre el mar -a qué profundidad viven dichas especies, océanos en peligro para crear conciencia...-, lo que facilita su desarrollo en ciencias naturales. El de educación física relata las historias de cincuenta deportistas relevantes desde 1800 hasta nuestros días. En educación artística aprenderán los pasos a seguir para dibujar poniendo en práctica la técnica del dibujo y, finalmente, a través de la música acercamos al disfrute del entorno marino.

Fase 4. Estrategias de visibilización del PLC.

Finalmente, en esta última fase se ha seleccionado un libro y se ha planificado un taller tomando como referencia uno de los métodos globalizadores que propone Zabala (1995), más concretamente el método de investigación basado en el libro El gran libro del mar haciendo uso de su epitexto -al que se puede acceder en la fase anterior haciendo clic en el enlace correspondiente al área de Ciencias Naturales-. Este taller - Anexo 1: Tabla 4- está dirigido a los alumnos que cursan sexto de Educación Primaria, teniendo en cuenta además que una de las asignaturas de este curso es Cultura y Práctica Digital. No obstante, se propone su dinamización como modelo dentro de un proyecto superior - Tabla 5- que pudiera desarrollarse con cualquier otro libro informacional.

Tabla 5

Distribución de sesiones del libro

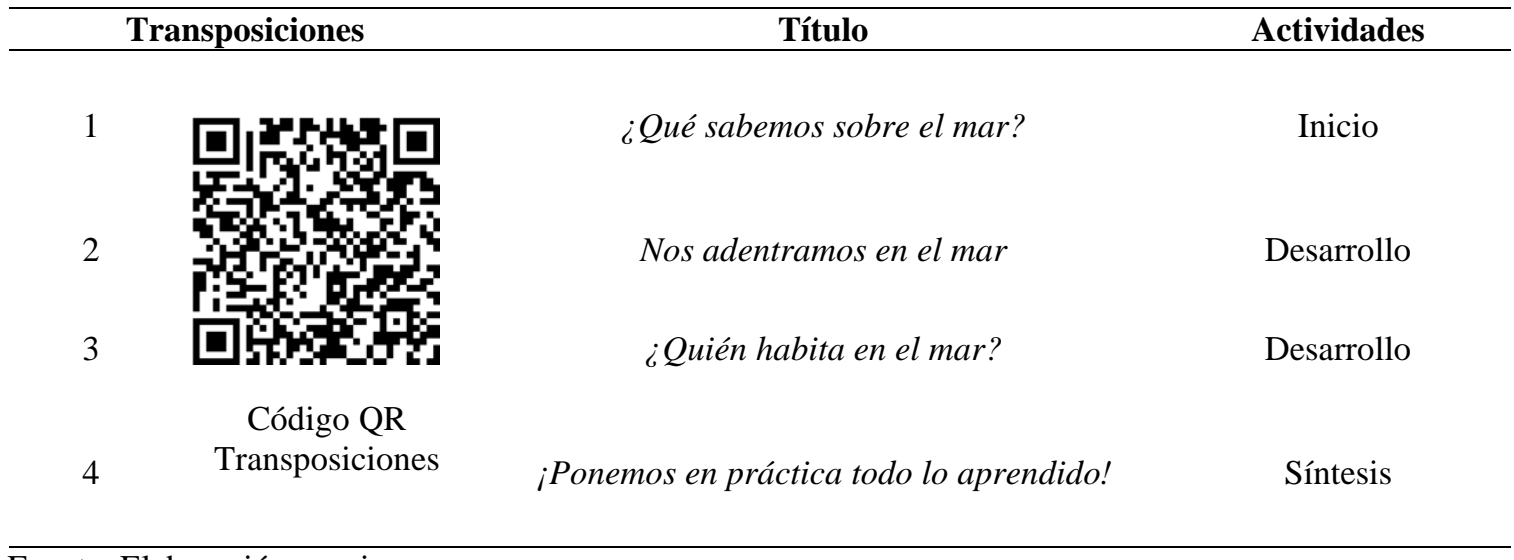

Fuente: Elaboración propia.

La intención se centra en ofrecer estrategias para el desarrollo de la competencia informacional y mediática, mediante un modelo de actuación a nivel de aula con el fin de integrar la lectura y contribuir al desarrollo de la formación de lectores desde los libros ilustrados de no ficción. De esta manera estaremos promoviendo el aprendizaje y la interpretación de nuestro entorno más próximo partiendo de la curiosidad que nos ofrece este género.

\section{Conclusiones}

A modo de conclusión, cabe decir que el objetivo ha sido alcanzado y se ha dado respuesta a las cuestiones de investigación planteadas. Por ello, tanto el proceso seguido como los resultados obtenidos ayudarán a los destinarios principales de este estudio -los docentes- a introducir mejoras e incorporar las innovaciones surgidas de esta investigación en sus contextos de aula. Tal y como se ha podido

Espiral. Cuadernos del Profesorado | ISSN 1988-7701 | 2021, 14(29), 41-55 
comprobar, el estudio de las distintas editoriales de los libros ilustrados de no ficción es bastante extenso $\mathrm{y}$, en consecuencia, se considera una oportunidad para todo profesional de educación tener como referencia esta cartografía lectora - Tabla 2-, pues podrá localizar los libros apropiados con el fin de integrarlos en las diferentes áreas del currículo, tanto en AL como en ANL. Pues, además, como se ha indicado en la propuesta didáctica - Tabla 3-, estos libros, también denominados libros informacionales, ofrecen la posibilidad de desarrollar habilidades y estrategias de lectura crítica, al tiempo que amplían el conocimiento de las diferentes áreas de contenido y, de esta forma, prepararan a los estudiantes para el futuro contribuyendo al desarrollo de la alfabetización informática, combinando la lectura por placer con la lectura por información (Young, et al. 2007).

Por otro lado, se ha podido constatar el fuerte grado de influencia que ejerce la industria editorial sobre las opciones y selección de lecturas ya que en sus plataformas ofrecen una amplia selección de títulos que, además, vienen acompañados de recomendaciones, críticas y complementos multimodales en la red que repercuten en la selección de lecturas escolares adquiriendo el rol de mediación desde la propia editorial y los medios de comunicación que marcan las preferencias lectoras. De esta forma, no debemos obviar el peligro con el que nos podemos encontrar puesto que, como indican Dueñas y Tabernero (2013, p.70),

las decisiones sobre el canon se tienden a adoptar al margen del profesorado. Son las circunstancias comerciales, la atmósfera de cambio permanente que se ha establecido en el mundo editorial, quienes deciden en buena parte acerca de la selección y conveniencia de unos libros y otros.

Por ello, la formación crítica de los docentes tanto en su formación inicial como permanente (Trigo, 2016) se hace necesaria para recuperar su protagonismo.

Asimismo, se ha podido constatar que los epitextos virtuales públicos (booktrailers, reseñas, blogs...) que se recogen en este itinerario lector son el resultado de una búsqueda editorial y resultan de vital importancia para ahondar en las nuevas formas de promoción y mediación de los libros informacionales. Es decir: se trata de un modo de promoción dirigido a los niños y jóvenes del siglo XXI, ya que el uso de recursos digitales les supone desenvolverse en su zona de confort y acceden a la información a través de la relación que se establece entre palabra, imagen y sonido. Por todo ello, el análisis de los epitextos editoriales es cada vez más necesario en la difusión del libro para el lector y para el mediador. Por lo tanto, podríamos cerrar nuestro estudio recogiendo las palabras de TaberneroSala (2016, p. 34): "Leemos para ser y los medios sociales forman parte de la esencia del lector del siglo XXI".

Esta investigación puede abrir nuevas líneas como generar el interés de los docentes por la formación de lectores críticos y competentes desde los libros ilustrados de no ficción. De esta forma, la creación de un espacio compartido, como una plataforma virtual que actúe de repositorio de referencia y portal virtual de encuentro para las personas interesadas en este tipo de libros (Romero Oliva, et al., 2021).

Contribución de cada Autor: conceptualización, metodología, revisión y supervisión, Manuel Francisco Romero Oliva; conceptualización, validación y análisis, Blanca Florido Zarazaga; conceptualización, metodología, revisión y supervisión, Hugo Heredia Ponce

Financiación: Esta investigación fue financiada por el proyecto del Proyecto Formar lectores en la sociedad digital desde el libro de no ficción (FL_SD_LNF) - Agencia estatal de investigación - Convocatorias 2018 proyectos de I+D de Generación de Conocimiento, RTI2018-093825-B-I00

Conflicto de Intereses: Las/os autoras/es declaran que no tienen conflicto de intereses.

\section{Referencias}

Andalucía. Instrucción 8, de 15 de junio de 2020, de la dirección general de ordenación y evaluación educativa, por la que se establecen aspectos de organización y funcionamiento para los centros que imparten Educación Primaria para el curso 2020/2021. 
Andalucía. Instrucciones de 24 de julio de 2013, de la dirección general de innovación educativa y formación del profesorado, sobre el tratamiento de la lectura para el desarrollo de la competencia en comunicación lingüística de los centros educativos públicos que imparten educación infantil, educación primaria y educación secundaria.

Ballester, J., \& Ibarra, N. (2016). La educación lectora, literaria y el libro en la era digital. Revista chilena de literatura, (94), 147-171. https://bit.ly/395terA

Bauman, Z. (2019). Acerca de lo leve y lo líquido. Nueva revista de política, cultura y arte, (170), 34-43.

Bronfenbrenner, U. (1987). La ecología del desarrollo humano. Ediciones Paidós.

Campoy, T., \& Gomes, E. (2009). Técnicas e instrumentos cualitativos de recogida de datos. En A. Pantoja (Ed.), Manual básico para la realización de tesinas, tesis y trabajos de investigación (pp. 273- 300). EOS.

Cordón, J.A. (2018). Combates por el libro: inconclusa dialéctica del modelo digital. El profesional de la información, 27(3), 467-482. https://doi.org/10.3145/epi.2018.may.02

Dueñas, J.D., \& Tabernero, R. (2013). Los clásicos en el aula. Una propuesta: hipertextualidad y contexto histórico. Tejuelo, 16, 65-77.

Fabregat, S. (2016). El proyecto lingüístico de centro: aprender más y comunicar mejor desde todas las áreas. Aula de Secundaria, 19, 25-30.

Fabregat, S. (2018). La lectura y las habilidades comunicativas en el marco del Proyecto Lingüístico de Centro: una propuesta interdisciplinar de mejora de la competencia en comunicación lingüística. En M.I. de Vicente-Yagüe \& E. Jiménez (Eds.), Investigación e innovación en educación literaria (pp.229-240). Síntesis.

Gómez, A. (2013). El aprendizaje integrado de la lengua española y los contenidos de áreas no lingüísticas en los proyectos lingüísticos de centro. Porta Linguarum. Revista de Didáctica de las Lenguas Extranjeras, 20, 103-115. https://doi.org/10.30827/digibug.29564

Herrera, R. (2009). Magia de la letra viva: formar lectores en la escuela. Editorial Academia.

Lluch, G., Tabernero, R., \& Calvo, V. (2015). Epitextos virtuales públicos como herramientas para la difusión del libro". El profesional de la información, (24), 6, 797-804. https://doi.org/10.3145/epi.2015.nov.11

López, F. (2002). El análisis de contenido como método de investigación. XXI. Revista de Educación, 4, 167-179.

Lorenzo, N. (2015). Mejorar la comunicación para vivir y convivir en el mundo real. Cuadernos de Pedagogía, $458,22-27$.

Maina, M. (2018). Escuela y lectura: tensiones de la experiencia. Anuario digital de investigación educativa, (1), 210-219.

McMillan, J.H., \& Schumacher, S. (2006). Investigación educativa: Una introducción conceptual. Pearson Educación.

Rodríguez-Gómez, G. (1999). Proyecto Docente: Métodos de Investigación en Educación. Universidad de Cádiz.

Rodríguez-Gómez, G. (2011). Técnicas e instrumentos para la investigación. UNIR.

Rodríguez, G., Gil, J., \& García, E. (1999). Metodología de la investigación cualitativa. Aljibe.

Romero, M. (2009). Acceso a las competencias básicas educativas desde la lectoescritura. TABANQUE Revista Pedagógica, 22, 191-204.

Romero, M. F., \& Trigo, E. (2015). Herramientas para el éxito. Cuadernos de Pedagogía, 458, 1-5.

Romero, M.F., \& Trigo, E. (2018). Los proyectos lingüísticos de centro: Desarrollar la comprensión lectora en áreas no lingüísticas. Textos de Didáctica de la Lengua y de la Literatura, 79, 51-59.

Romero, M.F., Ambrós, A., \& Trujillo, F. (2020). Hábitos lectores de los adolescentes en un ecosistema llamado escuela: factores determinantes en estudiantes de educación secundaria. Investigaciones sobre la lectura, 13, 18- 34. https://doi.org/10.37132/isl.v0i13.295

Romero, M.F., Heredia, H., Trigo, E. \& Romero, C. (2021). Validación de parrilla de contenidos y desarrollo de plataforma digital de libros ilustrados de no ficción. Tejuelo, (34), en prensa.

Santos, I.C. (2017). Desarrollo curricular y recursos educativos en las bibliotecas escolares. Investigaciones Sobre Lectura, 7, 36-54.

Tabernero, R. (2011). Leer y mirar: claves para una poética de la recepción del libro-álbum y del libro ilustrado. Ensino Em Re-Vista, 18(1), 93-109. 
Tabernero-Sala, R. (2016). Los epitextos virtuales en la difusión del libro infantil: Hacia una poética del booktrailer. Un modelo de análisis. Ocnos, 15(2), 21-36. https://doi.org/10.18239/ocnos_2016.15.2.1125

Trigo, E. (2016). El papel de la formación inicial y permanente del profesorado para forjar lectores. Revista de Estudios Socieoeducativos, (4), 66-84.

Trigo, E., Romero, M.F., \& García, A. (2019). Las voces de los agentes dinamizadores en la implementación de un PLC como clave para la transformación de un centro educativo. Tejuelo, (30), 37-72. https://doi.org/10.17398/1988-8430.30.37

Trilla, J. (2005). La idea de ciudad educadora y escuela. Educación y ciudad (7), 73-106.

Trujillo, F. (2010). La competencia en comunicación lingüística como proyecto de centro: retos, responsabilidades y ejemplificaciones. Lenguaje y Textos, 32, 35-40.

Trujillo, F., \& Rubio, R. (2014). El PLC como respuesta sistémica al reto de la competencia comunicativa en entornos educativos formales: propuesta de análisis de casos. Lenguaje y Textos, (39), 29-38.

Vázquez, R., \& Angulo, F. (2003). Introducción a los estudios de casos: Los primeros contactos con la investigación etnográfica. Aljibe.

Young, T. A. Moss, B., \& Cornwell, L. (2007). The classroom library: A place for nonfiction, nonfiction in its place. Reading Horizons, 48(3), 1-18.

Zabala, A. (1995). La práctica educativa. Cómo enseñar. Graó.

Zabala, A., \& Arnau, L. (2014). Métodos para la enseñanza de las competencias. Graó. 


\section{Anexo I}

Tabla 5

Taller basado en el método de investigación del medio

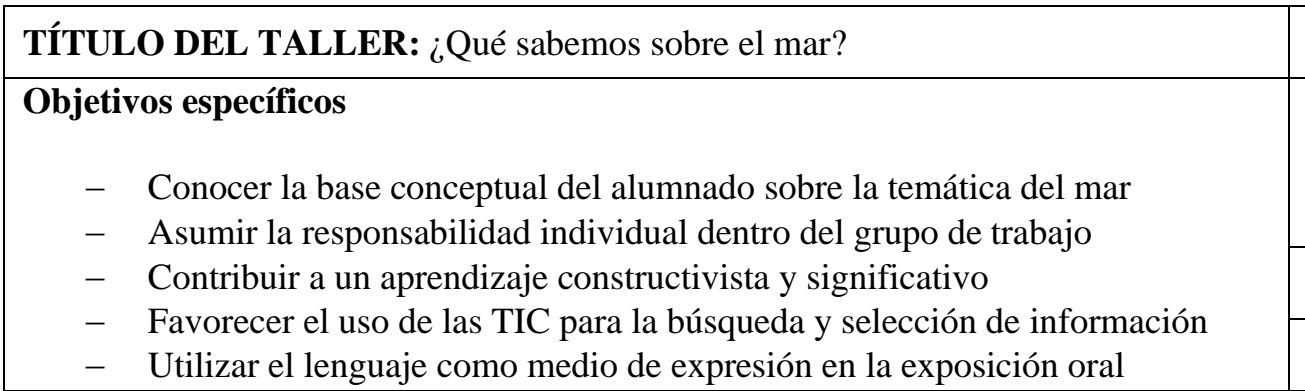

Descripción de las rutinas de la sesión
Contenidos (materias)

CCNN: $3.6-3.7-3.11-3.12$

CCSS: 2.10

LCYL: 2.1-2.4-2.6-3.1

CYPD: 3.1

Competencias clave

CCL, CAA, CSYC, SEIP, CD, CEC

Dinámicas y espacios

En primer lugar, se proyectará el epitexto de este libro (https://bit.ly/32kRa7U), en este caso una reseña, y se llegará a su comprensión siguiendo el proceso según Romero (2009): predecir el contenido mediante el título del texto, sintetizar el significado global, especificar la idea principal, interpretar el

1. Inicio de la sesión desde conocimientos previos contenido del texto y reflexionar a través de las siguientes cuestiones sobre el mar:

- ¿Qué significado tiene para vosotros el mar?

- ¿Cuidáis del mar?

- ¿Tenéis la oportunidad de disfrutarlo?

- ¿Consideráis que la sociedad está cuidando del mar?

Para ello, el docente deberá ser consciente de que será él quien se encargue de reconducir la temática en aquellos alumnos que, a pesar de estar participando, están alejados del argumento principal.

Se distribuirá al aula por grupos flexibles y heterogéneos para la posterior lectura de las páginas 56 y 57 del libro El gran libro

2. Presentación del tema del mar: océanos en peligro y plástico en el mar. Para profundizar aún más sobre los peligros del mar se proyectará el reportaje: contaminación marina: causas y consecuencias.

Aula

Se llevará a cabo la técnica del puzzle de Aronson con el fin de que investiguen sobre las causas que provocan un mayor impacto en el ecosistema marino. Para ello, las cinco causas seleccionadas serán: sobrepesca, productos químicos, calentamiento global, vertido de plásticos y plaguicidas/herbicidas.

Para poder formular conclusiones que realmente sean

3. Desarrollo de actividades verdaderas resulta ineludible hacer uso de medios adecuados y hacerlo de forma rigurosa. Por este motivo, el docente facilitará páginas web fiables, tales como: artículos, documentos, noticias, reportajes, enciclopedias digitales, etc.

Cada grupo base hará una selección de la información más relevante para responder a estas cuestiones en relación con el peligro atribuido: qué es, causas, consecuencias y soluciones. Los resultados se incluirán en el portfolio que, además de dejar constancia del trabajo realizado, será el soporte básico de estudio y el medio para sistematizar el recuerdo. 


\begin{tabular}{|c|c|c|c|}
\hline \multirow[b]{2}{*}{$\begin{array}{l}\text { 4. Cierre de la } \\
\text { sesión y } \\
\text { anticipación } \\
\text { de la siguiente } \\
\text { sesión }\end{array}$} & \multirow{2}{*}{\multicolumn{2}{|c|}{$\begin{array}{l}\text { Cada equipo de trabajo a través de diferentes técnicas } \\
\text { expresivas y de comunicación expondrá al grupo-clase la } \\
\text { información recogida en el porfolio. Para enlazar esta sesión } \\
\text { con la siguiente se les planteará la siguiente pregunta: } \\
\text { ¿Estos peligros afectan por igual a todos los animales que } \\
\text { habitan en el mar? }\end{array}$}} & \multirow{5}{*}{$\begin{array}{l}\text { Aula con mesas individuales que } \\
\text { pueden moverse para organizar } \\
\text { equipos de aprendizaje. } \\
\text { Conexión a internet. } \\
\text { Proyector y altavoces. } \\
\text { Portátiles. }\end{array}$} \\
\hline & & & \\
\hline \multicolumn{2}{|c|}{ Procedimientos de evaluación } & Instrumentos de evaluación & \\
\hline \multicolumn{2}{|c|}{$\begin{array}{l}\text { Actividad cooperativa } \\
\text { Exposición resultados }\end{array}$} & $\begin{array}{c}\text { Portfolio } \\
\text { Observación en aula }\end{array}$ & \\
\hline $\begin{array}{l}\text { Actividades } \\
-\quad \text { Lectura } \\
\text { información } \\
\text { porfolios s } \\
\text { peligros de } \\
\text { supervisa, a }\end{array}$ & $\begin{array}{l}\text { de refuerzo } \\
\text { autada de la } \\
\text { recogida en los } \\
\text { bre los distintos } \\
\text { mar. El docente } \\
\text { uda y corrige. }\end{array}$ & $\begin{array}{l}\text { Actividades de ampliación } \\
\text { - Lectura de la contaminación } \\
\text { marina en nuestras playas más } \\
\text { cercanas }\end{array}$ & \\
\hline
\end{tabular}

